\title{
Decreased Filamin b expression regulates trophoblastic cells invasion through ERK/MMP-9 pathway in pre-eclampsia
}

\author{
Jufeng Wei ${ }^{1}$, Yufen Fu2 ${ }^{2}$, Xiaoling Mao ${ }^{3}$, Yuhui Jing ${ }^{3}$, Jiangbo Guo ${ }^{3}$, Yuanhua Ye ${ }^{4}$ \\ ${ }^{1}$ Department of Obstetrics and Gynecology, Qingdao University, Department of Obstetrics, Qingdao Central Hospital, China \\ ${ }^{2}$ Department of Obstetrics, Zibo Maternity and Child Health Hospital, China \\ ${ }^{3}$ Department of Obstetrics, Qingdao Central Hospital, China \\ ${ }^{4}$ Department of Obstetrics, Affiliated Hospital of Qingdao University, China \\ ${ }^{*}$ Co-first author
}

\begin{abstract}
Objectives: The purpose of this study was to investigate the expression of Filamin b in the placental placenta of patients with early or late onset pre-eclampsia (PE) and its potential effects on the pathophysiology of the disease.

Methods and methods: Immunohistochemistry staining, western blot assays and real time PCR were used to detect the expression level of FLN-b. The expression levels of MMP-2, MMP-9 and ERK1/2 proteins from control and FLN-b-silenced JEG-3 cells were also detected by western blot and JEG-3 cell invasion.

Results: Compared with normal term pregnancies placentas, the FLN-b expression was significantly lower than that of women with PE, its level in late-onset PE is lower than in early-onset PE. In FLN-b-silenced JEG-3 cells, the protein levels of MMP-2, MMP-9 and phosphorylated ERK1/2 decreased markedly and the number of cells penetrating through the transwell chamber membrane is also greatly reduced.

Conclusions: Down-regulation of FLN-b inhibits the ERK/MMP-2 and MMP-9 pathways, leading to trophoblastic invasion disorders in the PE placenta.
\end{abstract}

Keywords: Filamin b; preeclampsia; placenta; invasion; ERK1/2

\section{INTRODUCTION}

Pre-eclampsia (PE), which affects $5 \%$ to $7 \%$ of the world's pregnant women each year, is a pregnancy-specific disease that is the leading cause of maternal and neonatal mortality and morbidity [1]. The disease is caused by a variety of factors and produces undesirable clinical features such as hypertension, proteinuria, inflammation, endothelial dysfunction, vasoconstriction and placental abnormalities [2,3]. PE can be divided into early-onset $P E$ ( $E P E$, $<34$ weeks of gestation) and late-onset $P E(L P E, \geq 34$ weeks of gestation) subtypes. Several pathological factors have been proposed to explain the development of $\mathrm{PE}$, including inadequate placental development, abnormal trophoblast invasion, vascular endothelial dysfunction, oxidative stress, immune maladaptation, exaggerated inflammation and genetic involvement. Despite years of ongoing clinical research, the etiology and pathogenesis of PE remains unclear.
Several key aspects of $P E$ can be studied in vitro, but the nature of the disease limits the utility of cell culture models. In particular, PE involves the changes in the behavior of fetal trophoblast cells, their interaction with the maternal endothelium, and the response of the maternal system to these vascular changes.

Filamins (FLNs) contain a series of high molecular weight cytoskeletal proteins that form a network linked to the surface of the cell membrane and play roles in the integration of cellular mechanics and signal transduction [4]. They consist of 3 isoforms in mammals: FLN-a, FLN-b and FLN-c. The functions of FLNs are complex as they regulate the activity of integrins and their ligand binding. FLNs also interact with a variety of cellular proteins, including transmembrane receptors, ion channels, signaling molecules and transcription factors that directly or indirectly regulate cellular responses and cellular movement $[5,6]$. FLN-a, for instance, has been clinically as- 
sociated with the aggressiveness of multiple cancers, including hepatic cholangiocarcinoma, pancreatic cancer, prostate cancer, metastatic breast cancer and high-grade astrocytoma [7-10]. FLN-b suppresses tumor growth and metastasis [11], enhances the invasion of cancer cells [12]. FLN-c expression is largely restricted to cardiac and skeletal muscle fibers and promotes their structural integrity [13].The aggressive invasion of trophoblastic cells in cases of PE is similar to the behavior of tumor cells. However, there has been a little research on the relationship between the FLN-b and the PE development, its expression levels of placental tissues in women between EPE and LPE, and its regulatory mechanisms. Proteins of the matrix metalloproteinase (MMP) family are involved in the breakdown of extracellular matrix in normal physiological processes, Gelatinase-A (MMP-2) and gelatinase-B (MMP-9) participates in S1P-evoked follicular ML-1 thyroid cancer cell invasion [14]. The MAPK signaling pathway pathway's general structure includes a small G protein (RAS) and three protein kinases (RAF, MEK, ERK). The starting point for this pathway is the binding of ligand to a transmembrane protein, a receptor tyrosine kinase (RTK). The resulting signaling cascade culminates with translocation of extracellular signal-regulated kinase ERK (mitogen-activated protein kinase, MAPK) to the nucleus, where ERK activates transcription factors that result in gene expression [15]. Activation or suppression of intracellular signaling via the MAPK family has been linked to expression of MMP in experimental models, MMP-2 mRNA expression ( $P=0.048)$, protein expression $(P=0.046)$ and gelatinolytic activity $(P=0.039)$ was positively correlated with ERK phosphorylative activity. MMP-2 activity $(P=0.017)$ and MMP-9 protein expression was also correlated with $\mathrm{p} 38$ activity and phosphorylation of p38 $(P=0.046)[16]$. We investigated the expression of FLN-b in placentas of the patients with EPE and LPE in addition to its pathophysiological effects on PE regarding MMP2, MMP9 and MAPK signaling pathway and phospho-ERK (p-ERK).

\section{MATERIAL AND METHODS}

\section{Patient information and placenta collection}

A written informed consent was obtained from all patients and the study protocol was approved by the ethics committee of the Affiliated Hospital of Qingdao University.
A total of 100 individuals, recruited from September 2014 to March 2015, were grouped as: 1. The premature delivery group (PD, $n=20$ ) included women aged 24-37 years with a gestational age $<37$ weeks without other complications; 2 . The normal singleton term pregnancies group $(S T P, n=20) ; 3$. The early gestation group $(E G, n=20)$, included healthy women undergoing legal abortion for nonmedical reasons; 4. The Preeclampsia group (PE), included 40 women with $P E$ aged 25-44 years. The diagnosis of the PE was based on clinical evidence. According to onset time as defined in the introduction above, they were further divided into two subgroups: EPE $(n=20)$, and LPE $(n=20)$ (Tab. 1). Smoking, women with chronic hypertension, alcohol consumption, women with eclampsia or gestational diabetes, and women with multiple pregnancies were excluded from the study.

The placentas were collected immediately after vaginal or Cesarean section delivery under aseptic conditions. After removing the decidual layer, the placenta tissues $\left(1.0 \mathrm{~cm}^{3}\right.$ each $\left.X 3\right)$ were washed 5 times in ice cold phosphate-buffered saline (PBS) to remove blood.

\section{Cell line}

The JEG-3 cells was acquired from the cell bank at the Chinese Academy of Sciences (Shanghai, P. R. China) and cultured in minimum essential medium (MEM, Gibco,USA), containing $10 \%(\mathrm{v} / \mathrm{v})$ fetal bovine serum (HyClone/GE Healthcare, Chicago, IL, USA), and $1 \%$ penicillin/streptomycin (Gibco), incubated at $37^{\circ} \mathrm{C}$ in a humidified atmosphere of $5 \% \mathrm{CO}_{2}$. Cells $\left(10^{5} / \mathrm{mL}\right)$ were seeded in 6-well plates for $30 \%-50 \%$ confluence at the time of transfection. Transfection using $50 \mathrm{nM}$ short interfering FLN-b-siRNA and incubated for $72 \mathrm{~h}$ to suppress native FLN-b expression was performed according to the manufacturer's instructions (Ribobio Life Technologies, Guangzhou, P. R. China), control cells were treated with the same volume of sterile PBS.

\section{Immunohistochemistry (IHC) staining}

The streptavidin-biotin-peroxidase complex method was used for IHC staining for all paraffin-embedded placental tissues. Sections (5-mm thick) were prepared and incubated with a primary antibody to FLN-b (1:150, Rabbit anti human mono-

\begin{tabular}{|l|l|l|l|l|l|l|}
\hline \multicolumn{2}{|l|}{ Table 1. The demographic and clinical characteristics of the five groups (Mean \pm SD) } \\
\hline Characteristics & $\begin{array}{l}\text { Premature } \\
\text { delivery }\end{array}$ & $\begin{array}{l}\text { Normal singleton } \\
\text { term pregnancies }\end{array}$ & $\begin{array}{l}\text { Early } \\
\text { gestation }\end{array}$ & $\begin{array}{l}\text { Early } \\
\text { onset PE }\end{array}$ & $\begin{array}{l}\text { Late } \\
\text { onset PE }\end{array}$ \\
\hline Maternal [years] & $27.14 \pm 2.96$ & $28.52 \pm 3.85$ & $27.82 \pm 2.43$ & $29.43 \pm 3.69$ & $29.32 \pm 2.92$ \\
\hline Times of gravidity & $2.17 \pm 0.98$ & $3.01 \pm 1.84$ & $2.32 \pm 1.08$ & $2.85 \pm 1.57$ & $2.69 \pm 1.32$ \\
\hline Gestational age [weeks] & $32.58 \pm 3.36^{*}$ & $38.73 \pm 4.28$ & $8.5 \pm 1.32^{*}$ & $33.49 \pm 2.83^{*}$ & $37.95 \pm 2.66$ \\
\hline Systolic blood pressure $[\mathrm{mmHg}]$ & $120.65 \pm 17.47$ & $123.69 \pm 14.70$ & $128.53 \pm 12.44$ & $153.39 \pm 13.72^{*}$ & $158.74 \pm 18.84^{*}$ & $<0.1481$ \\
\hline Diastolic blood pressure $[\mathrm{mmHg}]$ & $71.95 \pm 6.72$ & $73.41 \pm 7.85$ & $76.49 \pm 8.55$ & $113.58 \pm 9.8^{*}$ & $103.74 \pm 13.79^{*}$ & $<0.001$ \\
\hline
\end{tabular}


clonal antibody; Sigma-Aldrich, St Louis, MO, USA) overnight at $4^{\circ} \mathrm{C}$, then washed in PBS three times for 5 min each, incubated with a secondary antibody (Gene Tech, Shanghai, China) for $30 \mathrm{~min}$ at $37^{\circ} \mathrm{C}$. The sections were washed again three times with PBS. 3, 3'aminobenzidine (CW-BIO, Beijing, P. R. China) was used as the chromogen and hematoxylin (Sigma-Aldrich) was used as a nuclear counterstain.

\section{RT-qPCR}

The expression levels of $F L N b$ mRNA were detected using RT-qPCR. Total RNA was extracted from placental tissues using TRIzol protocol (Invitrogen Life Sciences, Carlsbad, CA, USA) and purified according to the manufacturer's instructions. $2 \mu \mathrm{g}$ of RNA were reverse transcribed in a $20-\mathrm{mL}$ reaction volume containing Superscript II reverse transcriptase (Invitrogen Life Sciences), incubated for $50 \mathrm{~min}$ at $42^{\circ} \mathrm{C}$, then for $15 \mathrm{~min}$ at $72^{\circ} \mathrm{C}$. The qPCR was performed using TaKaRa SYBR Premix Ex Taq Il kits (TaKaRa, Otsu, Japan) on an ABI Prism 7500 Real-Time PCR System (Applied Biosystems, Foster City, CA, USA) in triplicate in $25-\mu \mathrm{L}$ volumes: Aliquots of $2 \mathrm{~mL}$ of CDNA were mixed with $1.6 \mathrm{~mL}$ primers, $10 \mathrm{~mL}$ SYBR Premix Ex Taq II and $0.4 \mu \mathrm{L}$ ROX Reference Dye II. The PCR program was initiated at $95^{\circ} \mathrm{C}$ for $30 \mathrm{~s}$, followed by 40 cycles at $95^{\circ} \mathrm{C}$ for $5 \mathrm{~s}$ and annealing and extension at $60^{\circ} \mathrm{C}$ for $34 \mathrm{~s}$. A melting curve for primer validation and a template standard curve were generated to confirm template-independent amplification. Relative mRNA levels were calculated by using the 2-DDCt method against the normalized $b$-actin. The PCR primers used were: FLN-b: Forward, 5'-CAAATAACGGACCAAGAAGGA-3'; Reverse, 5'-C CGATAAGGAGAAAGTGGGATGT-3' b-actin: Forward, 5'-ATAGTTGCGTTACACCCTTTCTTG-3 Reverse, 5'-TCACCTTCACCGTTCCAGTTT-3'.

\section{Western blotting}

Placental tissues: the fresh frozen tissues were homogenized in $20 \mathrm{mM}$ Tris ( $\mathrm{pH} 7.4$ ), $137 \mathrm{mM} \mathrm{NaCl}, 2$ mM ethylenediaminetetraacetic acid (EDTA), $1 \mathrm{mM}$ phenylmethanesulfonyl fluoride (PMSF), 1\% Triton-X 100, 20 mM leupeptin, 10\% glycerol, $0.1 \%$ sodium dodecyl sulfate (SDS), $0.5 \%$ deoxycholate and $5 \mathrm{mg} / \mathrm{mL}$ aprotinin.

JEG-3 cells: JEG-3 cells were washed in PBS for 3 times. Proteins from tissues and JEG-3 cells were extracted using RIPA/PMSF (100:1) solution according to the manufacturer's instructions. $50 \mu \mathrm{g}$ protein was electro-separated on a 10\% SDS-PAGE gel and electro-transferred onto a polyvinylidene difluoride membrane (Millipore, Bedford, MA, USA) for Western blotting. After being blocked with 5\% skim milk for $1 \mathrm{~h}$ at room temperature, the PVDF membranes were incubated sequentially with primary antibodies (1: 2000, Abcam, Cambridge, MA, USA) against FLN-b, MMP2, MMP9, ERK1/2, PERK, and GAPDH (1:2500; Abcam) overnight at $4^{\circ} \mathrm{C}$, and then washed three times for 10 min each by $1 \times$ TBST (Tris-Buffered Saline with Tween 20). A subsequent incubation with a goat anti-rabbit HRP antibody (Abcam) was carried out for $1 \mathrm{~h}$ at room temperature in $5 \%$ skim milk, and three washes with TBST were carried out. Finally, enhanced chemiluminescence detection substrate was used to process the PVDF membranes (Pierce Chemical Co., Rockford, IL, USA). The image was analyzed using ChemiScope mini chemiluminescence meter to calculate the optical density of the target and the internal control band for the protein expression. Protein expression = integral optical density value of target protein/integral optical density value of GAPDH.

\section{Transwell assay}

Cell invasion assays were performed using transwell chambers precoated with Matrigel (BD Biosciences, Franklin Lakes, NJ, USA). The lower chamber contained MEM with 10\% FBS. The JEG-3 cells were pre-cultured with or without FLN-b siRNA ( $50 \mathrm{nM}$ ) about $72 \mathrm{~h}$. Then they were washed three times with PBS for $10 \mathrm{~min}$ and suspended in MEM, added $200 \mathrm{~mL}$ cell suspension $(1 \times 105$ cells $/ \mathrm{mL})$ to the upper chamber and incubated for $24 \mathrm{~h}$ at $37^{\circ} \mathrm{C}$. The upper chamber cells that did not invade through the membrane were wiped out using a sterile cotton-tipped swab. The filters were fixed in methanol and stained with 4', 6-diamidino-2-phenylindole (DAPI). The numbers of invasived cells with nuclei stained DAPI were counted under fluorescence microscopy.

\section{Statistical analysis}

At least three replications were done for each experiment. Data (mean \pm SD) were analyzed by Student-Newman-Keuls multiple comparison tests and independent $t$ tests using Graph-Pad Prism software (v. 5; La Jolla, CA, USA). Differences at $p<0.05$ were considered statistically significant.

\section{RESULTS}

\section{Localization and expression of FLN-b in placentas}

IHC showed (Fig. 1A) FLN-b was mainly expressed in the cytoplasm of trophoblasts and placental capillary endothelial cells. FLN-b expression in the placenta collected during PD, STP and EG was significantly higher, but it was declined in the placenta of PE (Fig. 1B).

RT-qPCR data (Fig.1C) indicated that the level of FLN-b mRNA in placentas of the EPE $(0.80 \pm 0.12)$ and LPE $(0.67 \pm 0.09)$ was significantly lower than that of the EG $(1.61 \pm 0.07)$, STP $(1.07 \pm 0.06)$, and PD $(1.32 \pm 0.09)$ groups $(p<0.05)$. The expression of FLN-b mRNA in the EPE placenta $(0.80 \pm 0.12)$ was higher than in LPE $(0.67 \pm 0.09)$.

The level of FLN-b protein in placentas of the LPE $(0.64 \pm 0.07)$ and of the EPE $(0.82 \pm 0.05)$ was significantly 


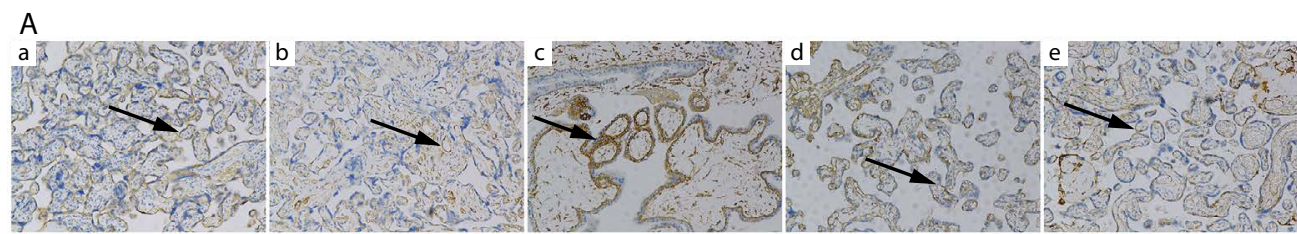

B

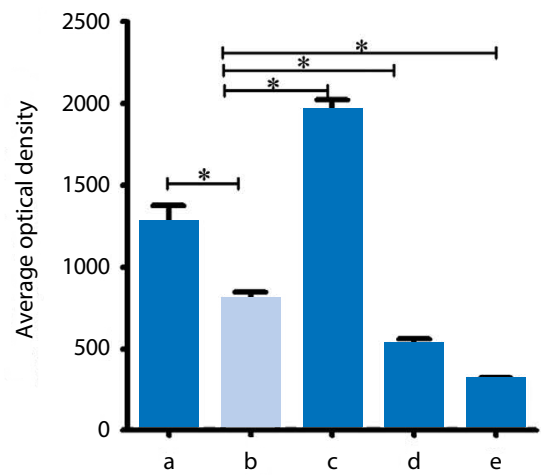

D

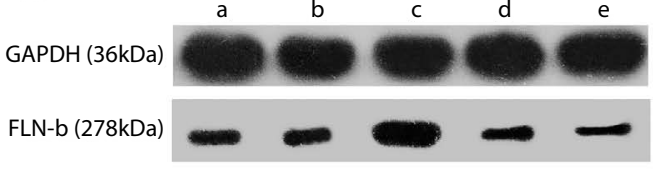

C

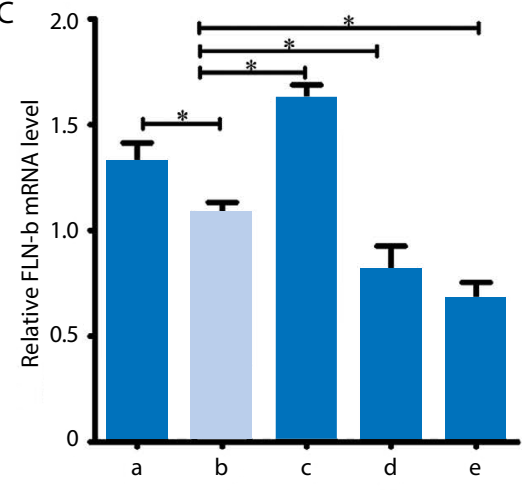

$\mathrm{E}$

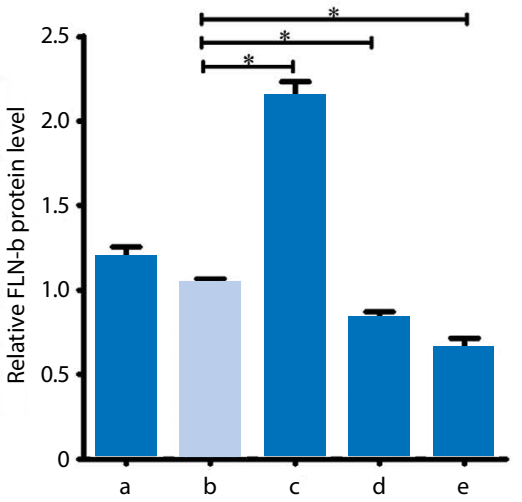

Figure 1. Expression of FLN-b in placental villi by immunohistochemistry $(400 \mathrm{X}) \mathrm{A}$ - The representative images of FLN-b expression in human placentas tissue: $\mathbf{a}$. Pretmature delivery; $\mathbf{b}$. Term delivery; $\mathbf{c}$. Early gestation; $\mathbf{d}$. Early onset PE; $\mathbf{e}$. Late onset PE; the black arrows indicated the positive staining; B — FLN-b average optical density assay of immunohistochemistry staining (Western bolting) and its statistical analysis $\left(\right.$ mean $\pm S D$ ); ${ }^{*} \mathrm{P}<0.05$

A

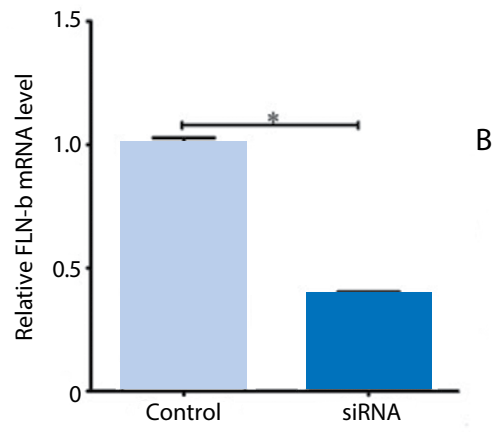

GAPDH (36kDa)

Control

SiRNA

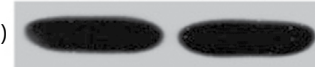

FLN-b (278kDa)

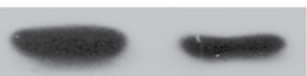

Figure 2. Relative expression of FLN-b in the control and FLN-b silenced JEG-3 cells. A - mRNA; B - FLN-b protein level

lower than that of the EG $(2.14 \pm 0.09)$, STP $(1.03 \pm 0.03)$, and PD groups $(1.18 \pm 0.08)(p<0.05)$. Although the expression of FLN-b decreased significantly in both EPE and LPE groups, the expression of FLN-b in the EPE placenta (Fig. 1D, $0.82 \pm 0.05)$ was significantly higher than in the LPE placenta (Fig. 1E, $0.64 \pm 0.07$ ).

\section{Depletion of FLN-b decreased the invasion rate in JEG-3 cells}

Figure $2 \mathrm{~A}$ presented that FLN-b mRNA relative expression level decreased in the FLN-b-silenced cells $(0.39 \pm 0.01)$ was compared with control cells $(1.00 \pm 0.03)$. Western blot analysis also confirmed that an FLN-b protein level in 

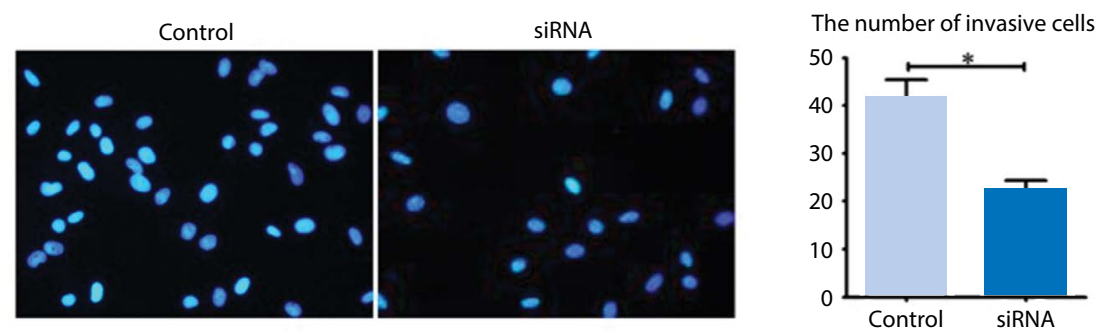

Figure 3. Effects of FLN-b on invasion of JEG-3cell. The number of invasive cells whose nuclei were stained was counted under fluorescence microscopic. The left pictures showed the nuclei of the invasive cells and the right picture showed statistical analysis of the number of invasive cells. ${ }^{*} \mathrm{P}<0.05$ versus control

FLN-b-silenced JEG-3 cells (53.42 \pm 0.96$)$ was significantly lower than that of the control cells (97.38 \pm 0.85$)$ (Fig. 2B). Transwell assay results showed that the FLN-b-silenced JEG-3 cell numbers $(22.00 \pm 2.31 \%)$ penetrated through the membrane were decreased significantly compared with the control $(41.33 \pm 4.10 \%)$ (Fig. 3$)$, suggesting that blocking FLN-b production reduced the invasive ability of in FLN-b-silenced JEG-3 cells.

\section{Depletion of FLN-b decreased MMP2, MMP9 and p-ERK expression levels in JEG-3 cells}

To further explore the effect of FLN-b and its concentration changes on JEG-3 cells, we measured the MMP-2, MMP-9 protein p-ERK and ERK levels by Western blotting. The expression levels of FLN-b $(61 \pm 0.01)$, MMP-2 (71.37 \pm 0.02$)$, MMP-9 (72.14 \pm 1.06$)$, p-ERK (72.41 \pm 1.58$)$ in FLN-b-silenced JEG-3 cells were significantly decreased as compared to the control group (Fig. $4 \mathrm{~B}$ to Fig. $4 \mathrm{E}, \mathrm{p}<0.05$ ), but there was no difference in the expression of total ERK between FLN-b-silenced JEG-3 cells $(75.83 \pm 2.27)$ and the control group (99.00 \pm 0.56 ). (Fig. $4 F, p>0.05)$ However, there was a decrease in the level of phosphorylated ERK1 $(44 \mathrm{kDa}$, Thr202/Tyr204) and of phosphorylated ERK2 (42kDa, Thr185/Tyr187) in FLN-b-silenced JEG-3 cells as compared to the control (Fig. 4E, P<0.05), indicating that MAPK signaling pathway has been implicated in these process.

\section{DISCUSSION}

PE is a systemic complication of pregnancy associated with high risks of preterm delivery, intrauterine growth restriction, placental abruption and perinatal mortality. Therefore, it is important to look for the reasons of PE. In this study, we explored the localization patterns, expression and potential roles of FLN-b in placentas from different groups and found that silencing FLN-b by siRNA reduced the invasive ability of JEG-3 cells and it was associated with a significant decrease in MMP2, MMP9 and p-ERK expression. Exogenous trophoblast invasion is associated with embryo implantation and placental formation. If this invasion is sup- pressed, the subsequent placental dysfunction can lead to various obstetric diseases, such as PE. The mechanisms of invasion of trophoblast cells are similar to those of tumor cells. Thus, FLN-b expression suppresses tumor growth and metastasis and enhances the invasion of cancer cells [11]. However, the function of FLN-b in of PE has barely been explored. Using IHC, RT-qPCR and western blotting techniques, we compared the distribution and expression of FLN-b in placentas derived from women with EG, STP, PD, EPE and LPE.

In this study JEG-3 cells, a trophoblast invasion model, was used to study to the role of FLN-b in PE assessed using cell motility and migration as well as the effect of FLN-b-silenced cells on trophoblastic invasion. Our results indicated that the invasion of FLN-b in siRNA-treated cells was reduced significantly. The invasion mechanism of trophoblast cells is complex and involves many molecules, including cell growth, adhesion, differentiation, and degradation of the extracellular matrix (ECM). The precise molecular mechanisms that regulate trophoblast invasion during gestation and its relationship to the placental development are largely unknown, but several proteinases, cytokines, and growth factors appear to be involved. MMPs are metal-dependent endopeptidases capable of degrading extracellular matrix. MMPs and their regulators, including tissue inhibitors of metalloproteinase (TIMPs), appear to play a critical role in mediating trophoblast invasion [17-20]. The degradation of ECM is thought to contribute to the invasion of trophoblast cells into the endometrium [21]. It has been reported that the key protein hydrolase in the trophoblast invasion is the MMP family. MMP-2 is the most important enzymes for the degradation of type IV collagen. The MMP-9 has been identified as a trophoblast-derived MMP [22].

By either directly modulating the actin cytoskeleton or scaffolding for other signaling molecules, FLNs are widely recognized as critical factor for the cell motility. FLN-a deficient human melanoma cells and FLN-b-/- MEFs show significantly reduced cell migration. FLN-b-/- tumor cells also displayed enhanced invasive capability in vitro [23]. 
A

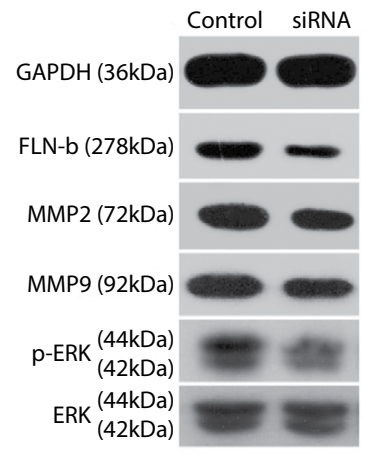

D

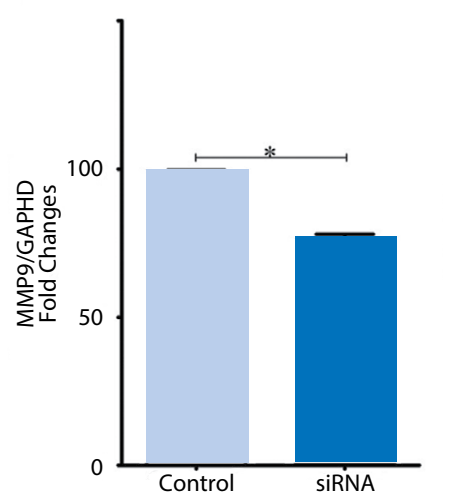

B

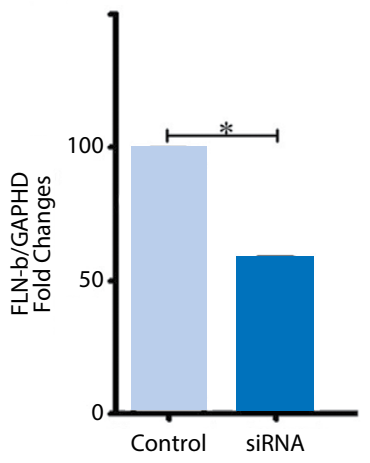

$\mathrm{E}$

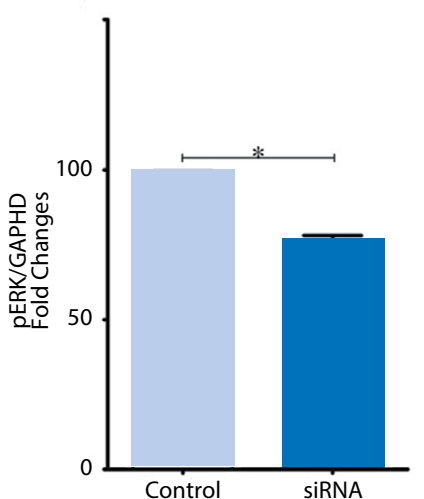

C

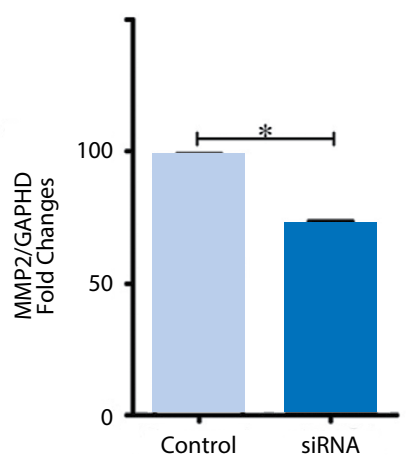

$\mathrm{F}$

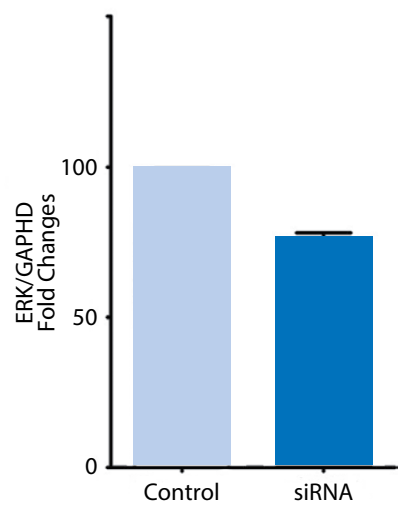

Figure 4. FLN-b, MMP2, MMP9 $p$-ERK and ERK protein expression level in the control and in the FLN-b silenced JEG-3 cells $\mathbf{A}$ - The protein expression of FLN-b, MMP-2, MMP-9, p-ERK and ERK assayed by Western blotting; B - The relative protein expression of FLN-b (mean \pm SD). ${ }^{*} \mathrm{P}<0.05 ; \mathbf{C}-$ The relative protein expression of MMP-2 (mean $\left.\pm S D\right)$. ${ }^{*} \mathrm{P}<0.05 ; \mathbf{D}$ - The relative protein expression of MMP-9 (mean \pm SD). ${ }^{*} \mathrm{P}<0.05 ; \mathbf{E}-$ The relative protein expression of $\mathrm{p}$-ERK (mean $\pm \mathrm{SD}$ ). ${ }^{*} \mathrm{P}<0.05 ; \mathbf{F}-$ The relative protein expression of ERK (mean \pm SD). $P>0.05$

However, Bandaru et al. found that the increased invasion capability in vitro and the enhanced metastasis in vivo of FLN-b-deficient tumor cells may be mediated by MMP-9 expression regulated by the MAPK / ERK cascade [24].

Our results showed that FLN-b was expressed in all of the normal term and PE groups. Most of the expression was located in the cytoplasm of trophoblastic and placental capillary endothelial cells. The IHC staining levels of FLN-b in the PE placentas were lower than that in the normal gestation placental tissues. These results are consistent with those of Bandaru et al. [11] which suggested a differential role of FLN-b in tumor progression may also be seen in $\mathrm{PE}$. In addition, the expression of FLN-b mRNA and protein levels were reduced in the placentas in the women with EPE as compared to LPE. The expression of FLN-b in EPE and LPE were lower than that of women with PD, STP and EG. The depletion of FLN-b was associated with the decreased MMP-2 and MMP-9 expression. The activation of MMP is mainly regulated by the ERK $1 / 2$ phosphorylation pathway [25]. We observed that the total ERK1/2 level had no discernible difference between the FLN-b-silenced cells and control groups, whereas the p-ERK1/2 level showed a significant decrease in the FLN-b-silenced cells. This indicated that the low expression of MMP-2 and MMP-9 with FLN-b depletion may attribute to the p-ERK1/2 signal transduction pathway and its downstream effect, resulting in the decreased invasion of trophoblastic cells. Thus, FLN-b is likely to be functioned through the $\mathrm{p}$-ERK1/2 signaling pathway to upregulate the MMP-2 and MMP-9 expression levels during trophoblast invasion.

\section{REFERENCES}

1. Cornelius DC. Preeclampsia: From Inflammation to Immunoregulation. Clin Med Insights Blood Disord. 2018; 11: 1179545X17752325, doi: 10.1177/1179545X17752325, indexed in Pubmed: 29371787.

2. Redman CW, Sargent IL. Latest advances in understanding preeclampsia. Science. 2005; 308(5728): 1592-1594, doi: 10.1126/science.1111726, indexed in Pubmed: 15947178.

3. Maynard SE, Venkatesha S, Thadhani R, et al. Soluble Fms-like tyrosine kinase 1 and endothelial dysfunction in the pathogenesis of preeclampsia. Pediatr Res. 2005; 57(5 Pt 2): 1R-7R, doi: 10.1203/01. PDR.0000159567.85157.B7, indexed in Pubmed: 15817508.

4. Stossel TP, Condeelis J, Cooley L, et al. Filamins as integrators of cell mechanics and signalling. Nat Rev Mol Cell Biol. 2001; 2(2): 138-145, doi: 10.1038/35052082, indexed in Pubmed: 11252955.

5. Zhou X, Borén J, Akyürek LM. Filamins in cardiovascular development. Trends Cardiovasc Med. 2007; 17(7): 222-229, doi: 10.1016/j. tcm.2007.08.001, indexed in Pubmed: 17936203.

6. Zhou AX, Hartwig JH, Akyürek LM. Filamins in cell signaling, transcription and organ development. Trends Cell Biol. 2010; 20(2): 113-123, doi: 10.1016/j.tcb.2009.12.001, indexed in Pubmed: 20061151.

7. Guedj N, Zhan Q, Perigny M, et al. Comparative protein expression profiles of hilar and peripheral hepatic cholangiocarcinomas. J Hepatol. 2009; 51(1): 93-101, doi: 10.1016/j.jhep.2009.03.017, indexed in Pubmed: 19446907. 
8. Li C, Yu S, Nakamura F, et al. Binding of pro-prion to filamin A disrupts cytoskeleton and correlates with poor prognosis in pancreatic cancer. J Clin Invest. 2009; 119(9): 2725-2736, doi: 10.1172/JCI39542, indexed in Pubmed: 19690385.

9. Bedolla RG, Wang Yu, Asuncion A, et al. Nuclear versus cytoplasmic localization of filamin A in prostate cancer: immunohistochemical correlation with metastases. Clin Cancer Res. 2009; 15(3): 788-796, doi: 10.1158/1078-0432.CCR-08-1402, indexed in Pubmed: 19188148.

10. Alper O, Stetler-Stevenson WG, Harris LN, et al. Novel anti-filamin-A antibody detects a secreted variant of filamin-A in plasma from patients with breast carcinoma and high-grade astrocytoma. Cancer Sci. 2009; 100(9): 1748-1756, doi: 10.1111/j.1349-7006.2009.01244.x, indexed in Pubmed: 19594548

11. Bandaru $S$, Zhou AX, Rouhi $P$, et al. Targeting filamin B induces tumor growth and metastasis via enhanced activity of matrix metalloproteinase-9 and secretion of VEGF-A. Oncogenesis. 2014; 3: e119, doi: 10.1038/oncsis.2014.33, indexed in Pubmed: 25244493.

12. Iguchi Y, Ishihara S, Uchida Y, et al. Filamin B Enhances the Invasiveness of Cancer Cells into 3D Collagen Matrices. Cell Struct Funct. 2015; 40(2): 61-67, doi: 10.1247/csf.15001, indexed in Pubmed: 25925610.

13. Thompson TG, Chan YM, Hack AA, et al. Filamin 2 (FLN2): A muscle-specific sarcoglycan interacting protein. J Cell Biol. 2000; 148(1): 115-126, indexed in Pubmed: 10629222.

14. Kalhori V, Törnquist K. MMP2 and MMP9 participate in S1P-induced invasion of follicular ML-1 thyroid cancer cells. Mol Cell Endocrinol. 2015; 404: 113-122, doi:10.1016/j.mce.2015.01.037, indexed in Pubmed: 25643979.

15. McCain J. The MAPK (ERK) Pathway: Investigational Combinations for the Treatment Of BRAF-Mutated Metastatic Melanoma. PT. 2013; 38(2): 96-108, indexed in Pubmed: 23599677.

16. Davidson B, Givant-Horwitz V, Lazarovici P, et al. Matrix metalloproteinases (MMP), EMMPRIN (extracellular matrix metalloproteinase inducer) and mitogen-activated protein kinases (MAPK): co-expression in metastatic serous ovarian carcinoma. Clin Exp Metastasis. 2003; 20(7): 621-631, indexed in Pubmed: 14669793.
17. Ferretti C, Bruni L, Dangles-Marie V, et al. Molecular circuits shared by placental and cancer cells, and their implications in the proliferative, invasive and migratory capacities of trophoblasts. Hum Reprod Update. 2007; 13(2): 121-141, doi: 10.1093/humupd/dml048, indexed in Pubmed: 17068222.

18. Cohen $M$, Bisch of $P$. Factors regulating trophoblast invasion. Gynecol Obstet Invest. 2007; 64(3): 126-130, doi: 10.1159/000101734, indexed in Pubmed: 17934306.

19. Husslein $H$, Haider $S$, Meinhardt $G$, et al. Expression, regulation and functional characterization of matrix metalloproteinase-3 of human trophoblast. Placenta. 2009; 30(3): 284-291, doi: 10.1016/j.placenta.2008.12.002, indexed in Pubmed: 19155066.

20. Xu B, Nakhla S, Makris A, et al. TNF-a inhibits trophoblast integration into endothelial cellular networks. Placenta. 2011; 32(3): 241-246, doi: 10.1016/j.placenta.2010.12.005, indexed in Pubmed: 21215448.

21. Saito $S$, Nakashima A. A review of the mechanism for poor placentation in early-onset preeclampsia: the role of autophagy in trophoblast invasion and vascular remodeling. J Reprod Immunol. 2014; 101-102: 80-88, doi: 10.1016/j.jri.2013.06.002, indexed in Pubmed: 23969229.

22. Zou J, Xu Li, Ju Y, et al. Cholesterol depletion induces ANTXR2-dependent activation of MMP-2 via ERK1/2 phosphorylation in neuroglioma U251 cell. Biochem Biophys Res Commun. 2014; 452(1): 186-190, doi: 10.1016/j.bbrc.2014.06.001, indexed in Pubmed: 24924630.

23. Zhou X, Tian F, Sandzén J, et al. Filamin B deficiency in mice results in skeletal malformations and impaired microvascular development. Proc Natl Acad Sci U S A. 2007; 104(10):3919-3924, doi: 10.1073/pnas.0608360104, indexed in Pubmed: 17360453.

24. Bandaru $S$, Zhou AX, Rouhi $P$, et al Targeting filamin $B$ induces tumo growth and metastasis via enhanced activity of matrix metalloproteinase-9 and secretion of VEGF-A. Oncogenesis. 2014; 3: e119, doi: 10.1038/oncsis.2014.33, indexed in Pubmed: 25244493.

25. Jia RZ, Rui C, Li JY, et al. CDX1 restricts the invasion of HTR-8/SVneo trophoblast cells by inhibiting MMP-9 expression. Placenta. 2014; 35(7):450-454, doi: 10.1016/j.placenta.2014.04.011, indexed in Pubmed: 248364 